\title{
B-Spline Solution for a Convection-Diffusion Equation
}

\author{
H. CAglaR ${ }^{a}$, N. CAGLAR ${ }^{b, *}$, M. Ozer $^{c}$ \\ ${ }^{a}$ Istanbul Kultur University, Department of Mathematics-Computer, Istanbul, Turkey \\ ${ }^{b}$ Istanbul Kultur University, Faculty of Economic and Administrative Science, Istanbul, Turkey \\ ${ }^{c}$ Istanbul Kultur University, Department of Physics, Istanbul, Turkey
}

This paper is concerned with the numerical solution of the convection diffusion problems. A family of B-spline methods has been considered for the numerical solution of the problems. The results showed that the present method is an applicable technique and approximates the exact solution.

DOI: $10.12693 /$ APhysPolA.125.548

PACS: 02.60.-x, 02.60.Lj

\section{Introduction}

Diffusion is one of the most important mechanisms in natural systems. It takes place in solids, liquids and gases. It can be applied in several problems such as heat flow through a medium or the transport of atoms, ions or molecules under a concentration gradient [1]. It is used not only in science and engineering but also in mathematical models in finance like the Black-Scholes equation [2] and many other applications. The convection diffusion equation describes the energy-loss mechanism by a combination of the wave and diffusion equations

$$
\frac{\partial u}{\partial t}+\alpha \frac{\partial u}{\partial x}=\beta \frac{\partial^{2} u}{\partial x^{2}}, \quad 0 \leq x \leq 1, \geq 0,
$$

to Eq. (1) we attach the initial condition and boundary conditions

$$
\begin{aligned}
& u(x, 0)=f(x), \quad 0 \leq x \leq 1, \\
& u(0, t)=g_{0}(t), \quad t \geq 0, \\
& u(1, t)=g_{1}(t), \quad t \geq 0 .
\end{aligned}
$$

This equation shows the wave equation and the heat equation (also called Fick's second law) for $\beta=0$ and $\alpha=0$, respectively. The former one conserves the energy and the latter dissipates the energy. Note that these losses are not too serious. It means that the coefficient $\alpha$ must be very small compared with the coefficient $\beta[3]$. In our previous work [4], the one-dimensional heat equation with a nonlocal initial condition is examined by using the third degree B-splines functions. In this paper, we have extended previous work on the convection diffusion equation using a family of B-spline methods. We have focused on some problems given in [5].

\section{The third-degree B-splines}

In this section, third-degree B-splines are used to construct numerical solutions to the convection-diffusion equations discussed in Sects. 3 and 4 . A detailed description of B-spline functions generated by subdivision can be found in [6].

\footnotetext{
*corresponding author; e-mail: ncaglar@iku.edu.tr
}

Consider equally-spaced knots of a partition : $a=x_{0}<$ $x_{1}<\ldots<x_{n}=b$ on $[a, b]$. Let $S_{3}[\pi]$ be the space of continuously-differentiable, piecewise, third-degree polynomials on $\pi$. That is, $S_{3}[\pi]$ is the space of third-degree splines on $\pi$. Consider the B-splines basis in $S_{3}[\pi]$. The third-degree B-splines are defined as:

$$
B_{0}(x)=\frac{1}{6 h^{3}}\left\{\begin{array}{l}
x^{3}, \quad 0 \leq x<h, \\
-3 x^{3}+12 h x^{2}-12 h^{2} x+4 h^{3}, \\
h \leq x<2 h, \\
3 x^{3}-24 h x^{2}+60 h^{2} x-44 h^{3}, \\
2 h \leq x<3 h, \\
-x^{3}+12 h x^{2}-48 h^{2} x+64 h^{3}, \\
3 h \leq x<4 h,
\end{array}\right.
$$$$
B_{i-1}(x)=B_{0}[x-(i-1) h], \quad i=2,3, \ldots
$$

To solve hyperbolic equation, $B_{i}, B_{i}^{\prime}$ and $B_{i}^{\prime \prime}$ evaluated at the nodal points are needed. Their coefficients are summarized in Table I.

Values of $B_{i}, B_{i}^{\prime}$ and $B_{i}^{\prime \prime}$.

TABLE I

\begin{tabular}{c|c|c|c|c|c}
\hline \hline & $x_{i}$ & $x_{i+1}$ & $x_{i+2}$ & $x_{i+3}$ & $x_{i+4}$ \\
\hline$B_{i}$ & 0 & $1 / 6$ & $4 / 6$ & $1 / 6$ & 0 \\
$B_{i}^{\prime}$ & 0 & $-3 / 6 h$ & $0 / 6 h$ & $3 / 6 h$ & 0 \\
$B_{i}^{\prime \prime}$ & 0 & $6 / 6 h^{2}$ & $-12 / 6 h^{2}$ & $6 / 6 h^{2}$ & 0
\end{tabular}

\section{B-spline solutions for the convection- -diffusion equation}

In this section a spline method for solving the convection-diffusion equation is outlined, which is based on the collocation approach [7]. Let

$$
S(x)=\sum_{j=-3}^{n-1} C_{j} B_{j}(x)
$$

be an approximate solution of Eq. (1), where $C_{i}$ are unknown real coefficients and $B_{j}(x)$ are third-degree B-spline functions. Let $x_{0}, x_{1} \ldots, x_{n}$ be $n+1$ grid points in the interval $[a, b]$, so that

$$
\begin{aligned}
& x_{i}=a+i h, \quad i=0,1, \ldots n ; \quad x_{0}=a, \quad x_{n}=b, \\
& \quad h=(b-a) / n .
\end{aligned}
$$


We consider the convection-diffusion Eq. (1). The difference schemes for this problem are considered as following:

$$
\frac{u_{i+1}-u_{i}}{\Delta t}+\alpha \frac{\partial u}{\partial x}=\beta \frac{\partial^{2} u}{\partial x^{2}},
$$

where $\Delta t=k$

$$
-k \beta u_{i+1}^{\prime \prime}+k \alpha u_{i+1}^{\prime}+u_{i+1}=u_{i}
$$

and the initial condition is given in $(2)$

$$
u(x, 0)=f(x)=u_{0},
$$

Substituting (9) in (8) then is obtained as follows:

$$
\begin{array}{ll}
t & =0+\Delta t, \quad-k \beta u_{1}^{\prime \prime}+k \alpha u_{1}^{\prime}+u_{1}=u_{0}, \\
t & =0+2 \Delta t, \quad-k \beta u_{2}^{\prime \prime}+k \alpha u_{2}^{\prime}+u_{2}=u_{1}, \\
\vdots & \vdots \\
t & =0+n \Delta t,
\end{array}
$$

The approximate solutions of Eqs. (10)-(12) are sought in the form of the B-spline functions, $S(x)$, it follows that:

$$
\begin{aligned}
& t=0+\Delta t, \quad-k \beta S_{1}^{\prime \prime}+k \alpha S_{1}^{\prime}+S_{1}=u_{0}, \\
& t=0+2 \Delta t, \quad-k \beta S_{2}^{\prime \prime}+k \alpha S_{2}^{\prime}+S_{2}=u_{1}, \\
& \vdots \quad \vdots \\
& t=0+n \Delta t, \quad-k \beta S_{n}^{\prime \prime}+k \alpha S_{n}^{\prime}+S_{n}=u_{n-1},
\end{aligned}
$$

and boundary conditions (3), (4) can be written as follows:

$$
\begin{array}{ll}
\sum_{j=-3}^{n-1} C_{j} B_{j}(0)=g_{0}(t) & \text { for } x=0, \\
\sum_{j=-3}^{n-1} C_{j} B_{j}(1)=g_{1}(t) & \text { for } x=1 .
\end{array}
$$

The spline solution of Eq. (13) with the boundary conditions is obtained by solving to the following matrix equation (see $[8,9]$ ). The value of spline functions at the knots $\left.x_{i}\right\}_{i=0}^{n}$ are determined using Table I. Then we can write in matrix-vector form as follows:

$$
A C=F,
$$

where

$$
\begin{aligned}
C & =\left[C_{-3}, C_{-2}, C_{-1}, \ldots, C_{n-3}, C_{n-2}, C_{n-1}\right]^{\mathrm{T}}, \\
F & =\left[g_{0}(k), f(0), f(h), f(2 h), \ldots, f((n-1) h), g_{1}(k)\right]^{\mathrm{T}},
\end{aligned}
$$

T denoting transpose.

The matrix $A$ can be written as

$$
A=\left[\begin{array}{ccccccc}
\frac{1}{6} & \frac{4}{6} & \frac{1}{6} & 0 & 0 & \ldots & 0 \\
\varphi_{1} & \varphi_{2} & \varphi_{3} & 0 & 0 & \ldots & 0 \\
0 & \varphi_{1} & \varphi_{2} & \varphi_{3} & 0 & \ldots & 0 \\
. & . & . & . & . & . & . \\
. & . & . & . & . & . & . \\
. & . & . & . & . & . & . \\
0 & 0 & \ldots & \varphi_{1} & \varphi_{2} & \varphi_{3} & 0 \\
0 & 0 & \ldots & 0 & \varphi_{1} & \varphi_{2} & \varphi_{3} \\
0 & 0 & \ldots & 0 & \frac{1}{6} & \frac{4}{6} & \frac{1}{6}
\end{array}\right] \text {, }
$$

where

$$
\begin{aligned}
\varphi_{1} & =-k \beta\left(\frac{6}{6 h^{2}}\right)+k \alpha\left(\frac{3}{6 h}\right)+\frac{1}{6}, \\
\varphi_{2} & =-k \beta\left(\frac{-12}{6 h^{2}}\right)+k \alpha\left(\frac{0}{6 h}\right)+\frac{4}{6}, \\
\varphi_{3} & =-k \beta\left(\frac{6}{6 h^{2}}\right)+k \alpha\left(\frac{-3}{6 h}\right)+\frac{1}{6} .
\end{aligned}
$$

It is easy to see that the same approximation is applied in the other Eqs. (14), (15).

\section{Numerical results}

In this section, the method discussed in Sects. 2 and 3 is tested on the following problems from the literature [5], and absolute errors in the analytical solutions are calculated. All computations were carried out using MATLAB 6.5.

Example 1. Consider problem(1)-(4) with the initial condition

$$
\begin{aligned}
& \frac{\partial u}{\partial t}+0.1 \frac{\partial u}{\partial x}=0.02 \frac{\partial^{2} u}{\partial x^{2}}, \quad 0 \leq x \leq 1, \geq 0, \\
& u(x, 0)=\mathrm{e}^{1.17712434446770 x}, \quad 0 \leq x \leq 1,
\end{aligned}
$$

and boundary conditions

$$
\begin{aligned}
& u(0, t)=\mathrm{e}^{-0.09 t}, \quad t \geq 0, \\
& u(1, t)=\mathrm{e}^{1.17712434446770-0.09 t}, \quad t \geq 0 .
\end{aligned}
$$

By using the procedure discussed in Sect. 3, we obtain the following spline solution for $u_{1}(x, k)$ :

$$
\begin{aligned}
& u(x, k)=9414 B_{-3}(x)+9985 B_{-2}(x)+10591 B_{-1}(x) \\
& \quad+11233 B_{0}(x)+11914 B_{1}(x)+12636 B_{2}(x) \\
& \quad+13402 B_{3}(x)+14214 B_{4}(x)+15076 B_{5}(x) \\
& \quad+15990 B_{6}(x)+16959 B_{7}(x)+17987 B_{8}(x) \\
& \quad+19078 B_{9}(x)+20234 B_{10}(x)+21461 B_{11}(x) \\
& \quad+22762 B_{12}(x)+24142 B_{13}(x)+25606 B_{14}(x) \\
& \quad+27158 B_{15}(x)+28804 B_{16}(x)+30550 B_{17}(x) \\
& \quad+32402 B_{18}(x)+34367 B_{19}(x) .
\end{aligned}
$$

The exact solution of this problem is $u(t, x)=$ $\mathrm{e}^{1.17712434446770 x-0.09 t}$. The observed maximum absolute errors for various values of $k$ and for a fixed value of $n=21$ are given in Table II. The numerical results are illustrated in Fig. 1.

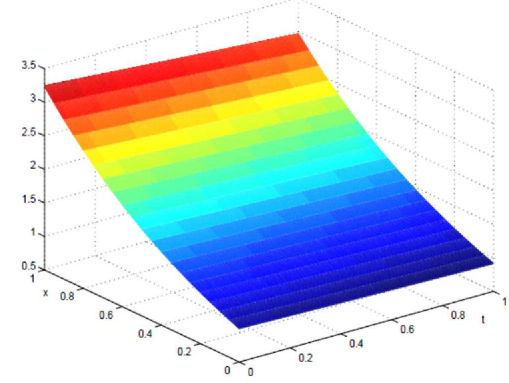

Fig. 1. Results for $\varepsilon=1, n=91, k=0.001$. 


\section{TABLE II}

The maximum absolute errors for problem 1 .

\begin{tabular}{c|c}
\hline \hline$n$ & 21 \\
\hline$k=0.1$ & $8.176749601 \times 10^{-4}$ \\
$k=0.01$ & $6.784132768 \times 10^{-5}$ \\
$k=0.001$ & $8.234124001 \times 10^{-6}$
\end{tabular}

Example 2. Consider the following problem,

$$
\begin{aligned}
& \frac{\partial u}{\partial t}+0.1 \frac{\partial u}{\partial x}=0.01 \frac{\partial^{2} u}{\partial x^{2}}, \quad 0 \leq x \leq 1, \geq 0, \\
& u(x, 0)=\mathrm{e}^{9 x}, \quad 0 \leq x \leq 1, \\
& u(0, t)=\mathrm{e}^{-0.09 t}, \quad t \geq 0 \\
& u(1, t)=\mathrm{e}^{9-0.09 t}, \quad t \geq 0,
\end{aligned}
$$

The exact solution of this problem is $u(t, x)=\mathrm{e}^{9 x-0.09 t}$. The observed maximum absolute errors for various values of $n$ and for a fixed value of $k=0.001$ are given in Table III. The numerical results are illustrated in Fig. 2.

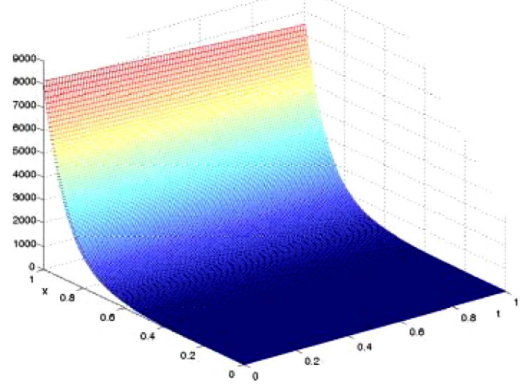

Fig. 2. Results for $n=311, k=0.01$.

\section{TABLE III}

The maximum absolute errors for problem 2.

\begin{tabular}{c|c}
\hline \hline$n$ & $k=0.01$ \\
\hline 111 & 0.756921814 \\
311 & 0.030124416 \\
411 & 0.014692091
\end{tabular}

Example 3. Consider the following problem:

$$
\begin{aligned}
& \frac{\partial u}{\partial t}+3.5 \frac{\partial u}{\partial x}=0.022 \frac{\partial^{2} u}{\partial x^{2}}, \quad 0 \leq x \leq 1, \geq 0, \\
& u(x, 0)=\mathrm{e}^{0.02854797991928 x}, \quad 0 \leq x \leq 1, \\
& u(0, t)=\mathrm{e}^{-0.09 t}, \quad t \geq 0, \\
& u(1, t)=\mathrm{e}^{0.02854797991928-0.09 t}, \quad t \geq 0,
\end{aligned}
$$

The exact solution of this problem is $(t, x)=$ $\mathrm{e}^{0.02854797991928 x-0.09 t}$. The observed maximum absolute errors for various values of $n$ and for a fixed value of $k=0.01$ are given in Table IV. The numerical results are illustrated in Fig. 3.

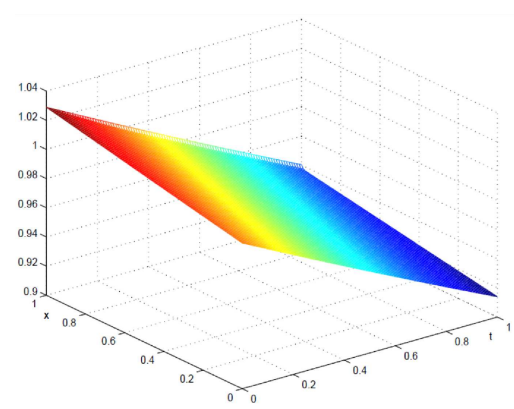

Fig. 3. Results for $n=111, k=0.01$.

\section{TABLE IV}

The maximum absolute errors for problem 3.

\begin{tabular}{c|c}
\hline \hline$n$ & $k=001$ \\
\hline 21 & 0.004306553 \\
61 & 0.003125393 \\
111 & 0.00269470629460
\end{tabular}

\section{Conclusions}

A family of B-spline methods has been considered for the numerical solution of the convection-diffusion equations. The third-degree B-spline has been tested on the convection-diffusion problems, and has tabulated the maximum absolute errors for different values of $n$ and $k$. As is evident from the numerical results, the present method approximates the exact solution very well. Also the numerical results are illustrated in figures. The implementation of the present method is more computational than other numerical techniques.

\section{References}

[1] R.J.D. Tilley, Understanding Solids: The Science of Materials, Wiley, London 2004, p. 203.

[2] P. Wilmott, S. Howison, J. Dewynne, The Mathematics of Financial Derivatives: A Student Introduction, Cambridge University Press, Cambridge 1995.

[3] H.J. Pain, The Physics of Vibrations and Waves, Wiley, 2005, p. 190.

[4] H. Caglar, M. Ozer, N. Caglar, Chaos Solitons \& Fractals 38, 1197 (2008).

[5] D.K. Salkuyeh, Appl. Math. Comput. 179, 79 (2006).

[6] C. de Boor, A Practical Guide to Splines, Springer Verlag, New York 1978.

[7] G.H. Golub, J.M. Ortega, Scientific Computing and Differential Equations, Academic Press, New York 1992.

[8] H. Caglar, N. Caglar, K. Elfaituri, Appl. Math. Comput. 175, 7279 (2006).

[9] N. Caglar, H. Caglar, Appl. Math. Comput. 182, 1509 (2006) 